\title{
Advances in Landslide Understanding
}

Landslide research covers an extremely wide range of aspects going from triggering mechanisms to the response of the unstable mass after failure, including transportation, deposition and interaction with protective structures. Advances in landslide research relies on accurate field data, comprehensive monitoring of laboratory experiments, especially those conducted in centrifuge, and improved numerical analyses. Integrating most of these aspects in a unified analysis of welldocumented case histories offers the opportunity to evaluate our current understanding and capabilities. Despite the general accessibility to numerical codes, theoretical analysis remain a most valuable source of knowledge and judgement.

The capabilities of the models and its soundness should be demonstrated. This is done, in this issue, by means of (a) simulating previously controlled and well-instrumented experiments and comparing numerical results with measurements and (b) by calibrating the model through laboratory tests and back-analysis of real cases. The calibrated model can also be used to explore its response to different conditions, not observed in the field.

Contributions to this issue offer excellent examples of most of the topics mentioned:

Choi, Ng and Cheung present experiments performed in the geotechnical centrifuge of the Hong Kong University of Science and Technology. The paper is a contribution of the HKUST group to understand the impact of granular flow against barriers. In this contribution, the focus is placed on the effect of the dynamic grain fragmentation on the impact force exerted on a barrier. The paper addresses the question of finding how the dynamic fragmentation of large particles, when impacting a rigid barrier, which is a process that dissipates energy, affect the resisting force required to the barrier. To do that, the dynamic fragmentation after impact is evaluated. A test protocol regarding grain size distribution of bi-dispersed granular flows is defined in order to reach practical conclusions. Grain sizes are characterized by normalizing the diameter of small particles by the diameter of large particles, which remains unchanged in all the tests performed. In conclusion, the dynamic fragmentation is a relevant mechanism to quantify the impact load exerted on a rigid structure. Unfortunately, as the authors mention, this is a first step and the application in practical 
engineering is limited because of the difficulties to quantify both flow composition and dynamic fragmentation of a granular flow in the field.

Kwan, Sze and Lam analyze also in this issue the landslide impact on barriers. They explore the barrier response by means of a 3D finite element code (LS-DYNA developed by Livermore Software Technology Corporation). Firstly, they investigate the response of a concrete wall with different types of cushioning materials subjected to spherical boulder impacts. The results of large-scale experiments reported in previous papers allow a previous calibration of the model. Once calibrated, the model is used to evaluate the problem and to obtain information difficult to measure in the field. In addition, parametric studies are carried out to a) evaluate the impact of boulders having different sizes and velocities and b) derive empirical and practical equations for calculating the impact forces. The impact forces are calculated using code functionalities, specifically designed to deal with contacts of different materials. The code incorporates appropriate constitutive models for the different cushioning materials analyzed.

The paper includes a second impact problem, namely the interaction between debris flow and steel flexible barriers. An arbitrary Lagrangian-Eulerian method is selected to simulate the large deformation of the debris flow. Beam elements model the structural components.. The model reproduces the coupled interaction between soil and structural elements. Two well-documented field experiments reported in the literature allows the verification of the model and shows its capabilities.

Su, Choi, Ng, Lam, Kwan, Wu, Huang and Zhang report on a new large-scale experiment of spherical boulder impact against a cushioning gabion basket made of recycled crushed glass. Results are compared with more conventional cushioning material such as rock fragments and cellular glass aggregates. The paper highlights the advantages of recycled crushed glass in terms of transmitted boulder impact loads to the wall.

An interesting example of the need to analyze specific phenomena in controlled scaled-test is the paper by Pagano, Reder and Rianna. They evaluate the effect of the vegetation, always difficult to quantify in the field, on the hydrological behaviour of a soil. To do that, they analyze the hydraulic response (suction and water content evolution) of a silty volcanic layer exposed to atmospheric conditions during three years in a highly instrumented tank of around $1 \mathrm{~m}^{3}$. The measurements and their theoretical interpretation are compared with the behavior observed under bare conditions. The conclusions serve to re-interpret a shallow landslide case-history which involved the soil tested 
in the tank. The model, previously used without including the effect of vegetation, is calibrated again including the new data. It is concluded that the vegetation affects the suction and water content profiles, which are directly related to the landslide response.

Two papers in this issue deal with the effect of rainfall on slopes, a common triggering cause of landslides. Measurements on scaled tests and field monitoring are crucial to understand the evolution of key variables affecting the slope stability. Damiano discusses, based on previous contributions, the effect of layering on water infiltration in shallow granular deposits. The discussion concentrates on the layered pyroclastic covers of Campania (Italy) where a flowslide occurred in 1999. The paper presents a joint analysis of the infiltration into the layered soil and the calculation of the safety factor, assuming a planar mechanism. The model is validated and calibrated by means of a flume test and the analysis of field monitoring data, recorded in the area of the 1999 flowslide during the period $2002-2007$.

The second paper dealing with rainfall-induced shallow landslide, by Balzano, Tarantino, Nicotera, Forte, De Falco and Santo, also deals with landslides occurred in Campania, Italy, in a layered profile. In this case, no scaled tests or field measurements are presented. A laboratory experimental program allowed the characterization of the materials involved in the two landslides analyzed. A finite element commercial program served to analyze the flow. The atmospheric conditions defining rainfall and evapo-transpiration are modelled for a period of four years before the failure events. Modeling a relative long period before failure is required to cancel the effect of the unknown initial conditions of the numerical model, which need to be imposed. The stability analysis is finally carried out in terms of the evolution of safety factor in time.

Three articles of this special issue present numerical modeling of landslides using the material point method (MPM). All of them focus on the post-failure behavior in order to evaluate the run-out stage. In particular, Yerro, Soga and Bray revisit a well-known case history: the Oso landslide in 2014. The paper describes a 2D Material Point Method (MPM) representation of the landslide. The main interest is to simulate, during landslide run-out, the interaction and overlapping among different mobilized materials. This is an interesting contribution to a well-known case, which was previously analyzed by depth-integrated numerical techniques. Previous publications, which describe laboratory and field tests, allowed the geotechnical characterization of the materials involved. The simulation of a previous landslide in the same place, eight years before the Oso event, allowed the calibration of the model. The model was ultimately adjusted by matching the distance actually 
traveled by the unstable mass. The paper concentrates on the post-failure behavior and run-out, leaving out of the analysis the triggering causes. The shear surfaces are pre-defined in the analysis by means a contact algorithm and the initiation of the failures are modelled artificially by reducing the undrained strength of the involved materials. Once the Oso landslide model was calibrated, it was used to perform a parametric analysis to evaluate the effect of different mechanisms (material fluidization due to water entrainment and the softening of the shear strength during motion).

The second paper using MPM for modeling is presented by $\mathbf{X u}$, Jin, Sun, Soga and Zhou. They simulate in three dimensions (3D) the Hongshiyan flow landslide (China), which was triggered by an earthquake, not simulated explicitly in the model. A constitutive model that combines a DruckerPrager plastic formulation and rheological relationships describes the landslide granular flow. The resulting model, described in detail, is a strain and strain rate-dependent model.

Before facing the field-scale test, the MPM code with the proposed constitutive model is first validated by simulating a granular pile collapse laboratory test, analyzed by means of particle image velocimetry (PIV). The results are very satisfactory and the case is used to evaluate the effect of model parameters. In particular, parameter $(\beta)$, related with the frictional material properties, determines the energy dissipation during motion and controls the flow process and the deposition stage.

The 3D geometry of the slope was generated by means of ArcGIS platform. Model parameters were estimated. The results matched the measured post-failure topography. The relevant parameter $\beta$ was identified by matching the maximum velocity reached by previous similar large-scale landslides.

Finally, Alvarado, Pinyol and Alonso explore the combined effect of strain rate and thermal interactions on landslide mobility. Rate effects on the angle of friction are able to explain long-term creeping records of real cases. On the other hand, the frictional work dissipated in heat may induce the rapid acceleration of the landslide due to the accumulation of thermally-induced pore water pressures and the subsequent reduction of effective frictional strength. The combination of the two effects are invoked in this paper to explain the observed behavior of a reactivated landslide due to a rapid drawdown (the partially submerged Canelles landslide, Spain).

A strain and strain rate dependent Mohr-Coulomb model describes the soft clay rock formation, responsible for the landslide. The increasing resistance of sliding, when creping rate increases, can explain that the landslide did not accelerate during its reactivation. After landslide triggering, 
thermal interaction acts by inducing excess pore water pressures. However, its effect is limited because of the increased strength available thanks to the higher landslide velocity. This effect is able to avoid the development of blow-up conditions. The landslide finally stops because of the positive effect of the evolved geometry. The numerical simulation presented in the paper demonstrates that the sliding resistance enhancement provided by shearing rate effects contributes significantly to reduce the risk of high, thermally-driven, landslide velocities.

The paper by Buss, Balz and Puzrin is a brilliant theoretical analysis of the slab type of failure of submarine landslides and the parallel spreading and ploughing failures at upper and lower elevations. The selected methodology (kinematic energy balance approach: KEB) is a remarkable tool which combines a relative simplicity and a flexibility to interpret complicated failure mechanisms. It all depends on the ability to use the method and the understanding of the physical aspects of the problem. The dynamic character of the method is a feature which allows analyzing the initiation and the propagation of the failure. The paper provides useful criteria to find the limiting loads leading to spread or ploughing failures in terms of the drop or heave of the soil surface. The paper shows how theoretical models may be close to observations. They are also useful to interpret and validate numerical analyses. Geotechnical engineers are probably not familiar with KEB. However, the method is a generalization of the common upper bound procedures in plasticity. In addition to its applicability to submarine landslides the method may be used to other landslide scenarios and, indeed, to other geotechnical situations.

This Special Issue includes papers originally presented to the First International Workshop of the Joint Technical Committee 1 on Natural Slopes and Landslides held in May 2017 in Barcelona. The following address http://congress.cimne.com/itc1/frontal/doc/Ebook.pdf provides an access to the book of extended abstracts submitted to the Workshop.

Contributors to the Workshop were invited to prepare and submit full manuscripts to the CGJ for refereeing and eventual publication acceptance.

The JTC1 was an initiative of the International Society for Soil Mechanics and Geotechnical Engineering, the International Society for Rock Mechanics and the International Association for Engineering Geology and the Environment. Its current Chairman is Professor Luciano Picarelli, Università degli Study della Campania, Naples. The Committee is active organizing Workshops on 
specific issues and promotes the preparation of reference documents on aspects related to landslide research and practice. The Committee is expected to advance the science and engineering practice behind any aspect concerning landslides.

The editors express their gratitude to the support provided by the JTC1 to carry forward the initiative of preparing a Special Issue with selected contributions. Deep thanks are extended to the Canadian Geotechnical Journal for its acceptance to launch this Special Issue and for the help received during the process of paper reviewing.

Barcelona, July $28^{\text {th }}, 2019$

Eduardo Alonso Núria M. Pinyol

Editors 